\title{
ERGODICITY OF AVALANCHE TRANSFORMATIONS
}

\author{
MANFRED DENKER AND ANA RODRIGUES
}

\begin{abstract}
In this paper we study dynamical systems of product type and some particular inducing scheme motivated by neural dynamics (called avalanche transformation). We derive the distribution of avalanche sizes and give sufficient conditions such that the avalanche transformation is ergodic. Moreover, we deduce a multivariate central limit theorem as a corollary.
\end{abstract}

In memoriam Wenbo V. Li (1963-2013)

Key words: Avalanche transformation, ergodicity, induced transformation, Cayley's theorem, central limit theorem AMS classification: 37A05, 37A50,60F05

\section{INTRODUCTION}

There are two basic methods in ergodic theory which have an application to neural dynamics. One of them is the one studied in this paper, the method of inducing on sets, which leads to avalanche transformations. The other method is a jump transformation which leads to a synchronization map.

The original motivation for this work comes from the study of avalanches in neural dynamics as studied by A. Levina (in [6]) and others (see the references there). Let us first recall this motivation following this reference. There are two types of cells in the central nervous system, one of them is called neuron, which communicate by sending and receiving electrical impulses. The cell membrane has built into it channels and ion pumps, letting potassium ions rushing out, and sodium ions flushing in. This process of exchange of potassium and sodium ions (once initiated) stops after a few milliseconds when repolarisation is achieved. The process needs an external activation for getting started.

The integrate and fire model to describe this phenomenon goes back to Lapicque in 1907 ([5]). It describes the time series of the potential of the cell membrane. It can be written as an ordinary differential equation of the form

$$
C_{m} \frac{d V(t)}{d t}=g_{l}\left(V_{\text {res }}-V(t)\right)+I(t)
$$

where $C_{m}$ is called the capacitance of the cell membrane, $V(t)$ is the voltage (potential) at time $t, V_{\text {res }}$ is the residual potential, $g_{l}$ is the leak conductivity of the cell

Date: March 23, 2016.

The research of MD was supported by the National Science Foundation grant DMS-1008538. The research of AR is supported by the Swedish Research Council (VR Grant 2010/5905). The authors would like to thanks the Göran Gustafsson Foundation UU/KTH for financial support.

${ }^{1} \mathrm{~A}$ few weeks before his sudden death on January 26, 2013 we received a comment on this paper which is reproduced at the end of this article. 
membrane and $I(t)$ is the current at time $t$. This time evolution of the potential of a neuron is interrupted if the potential reaches a certain threshold value (this is when the process of exchange of potassium and sodium ions starts). At this point it gives a fixed electrical impulse to each of its neighboring neurons. Thus other neurons' potential may reach the threshold, thus initiating an avalanche of firing neurons.

The model of Eurich, Herrmann and Enst ([4]) is a time discrete version of this equation and is formulated as a discrete time dynamical system by Levina ([6]). The increase of potential is modeled by the maps $\left(x_{1}, \ldots, x_{N}\right) \mapsto\left(x_{1}, \ldots, x_{i-1}, x_{i}+\right.$ $\left.\delta, x_{i+1}, \ldots, x_{N}\right)$ for some random choice of the index $i$ and by the map $\left(x_{1}, \ldots, x_{N}\right) \mapsto$ $\left(x_{1}+\alpha, \ldots, x_{N}+\alpha\right)$ for the avalanche action; all maps are considered modulo the treshold value and $N$ denotes the number of neurons in the system. It is known from [6] that Lebegue measure on a unique invariant set $\subset[0,1]^{N}$ is invariant, but it is unknown whether it is ergodic in case that $\alpha$ and $\delta$ are rationally independent.

Here we consider only one map, but the same avalanche algorithm as in the Eurich, Herrmann, Ernst model. Thus the present work is not meant to explain the features of Levina's model, it is mainly a toy model to study basic properties of the avalanche algorithm in connection with one transformation. Instead of using rotations as in [6] we are able to work with general measure preserving transformations, invertible or not and not necessarily the same for each neuron. We derive a general combinatorial formula for the distribution of the avalanche dynamics which is based on Cayley's theorem on the number of labeled trees with $N$ vertices. This result is different to the one in [4] but has some connection via expectations; a note on this expectation is added at the end which was contributed by Wenbo Li shortly before his sudden death. For our dynamics, when the maps are invertible, we can characterize ergodicity completely in Theorem 4.3 by the eigenvalues of the transformations. It shows that the ergodicity question of Levina is of different nature, since it implies that for the Levina model in the case of $\alpha=\delta$ we cannot have ergodicity unless $N=1$. This result is as well general, while our last result on the central limit theorem (as a basic result for data analysis) requires a sort of mixing in the sense that each transformation modeling the increase of the potential of a neuron satisfies the central limit theorem. This is often satisfied if each of these transformations has some mixing (or the processes generated by them).

The paper is organized as follows. In Section 2 we study a combinatorial question which arose in connection with the avalanche distribution of our avalanche model, i.e. the distribution of the number $A$ of firing neurons in an avalanche, which is related but not the same as the one used in [4] and in [6] as explained above. In particular, this investigation led us to a simple and elementary proof of Cayley's formula on the number of trees with $n$ vertices ${ }^{2}$.

In Section 3 we determine the avalanche distribution of $A$. We derive an explicit formula similar (but different) to the result in [4]. This leads to a different proof of the expectation of the distribution in [4], a result which originally was proved in [6]. This distribution was coined Abelian distribution and will appear as a note in [7].

\footnotetext{
${ }^{2}$ Acknowledgment: We would like to thank Ira Gessel and Wlodek Bryc for some helpful remarks concerning Section 2. This may be of independent interest in view of the many proofs which appeared for this theorem (see [8], [9]).
} 
In Section 4 we show that the avalanche dynamics is ergodic when the transformations $S_{i}$ modeling the neurons' potentials have different eigenvalues except $\lambda=1$. In fact this is shown by representing the avalanche transformation $S_{A}$, restricted to its wandering set, as an induced transformation under the product dynamics $S=S_{1} \times \ldots \times S_{N}$. Finally, we derive a central limit theorem for $S_{A}$ as a corollary from this representation.

\section{CAYley's Theorem}

In this section we prove an auxiliary result that will serve as a tool for the investigation of the avalanche transformation. In particular, it will be used when finding the avalanche size distribution.

In 1889, Cayley ([2]) showed that the number of labeled trees with $n$ distinguishable vertices is $n^{n-2}$. We begin giving an apparently new proof of this result. The article of Moon ([8]) lists ten different proofs. Renyi ([9]) gave another proof of this fact. As noticed in the beginning of the proof by Clarke ([3]), all trees labeled with $n+1$ points can be represented as rooted trees where an arbitrary chosen vertex is fixed as the root. Counting these trees by dividing the remaining $n$ vertices into $r$ subsets $V_{1}, \ldots, V_{r}$ of sizes $k_{1}, \ldots, k_{r}$ and letting $V_{l}$ denote the vertices at distance $l$ from the root (in the path lengths metric), one has $k_{l-1}^{k_{l}}$ choices to connect to the set $V_{l-1}$. Given the Cayley result we thus have a proof of the following Theorem 2.1. Conversely, we shall prove Theorem 2.1 by simple induction, which leads to a new proof of Cayley's Theorem as an obvious corollary. Let $\mathbb{N}$ denote the set of natural numbers $1,2, \ldots$. Define

$$
M_{r}(n)=\left\{\left(k_{1}, \ldots, k_{r}\right) \in \mathbb{N}^{r}: k_{1}+k_{2}+\ldots+k_{r}=n\right\} .
$$

Theorem 2.1. For each $n \in \mathbb{N}$,

$$
\sum_{r=1}^{n} \sum_{\left(k_{1}, \ldots, k_{r}\right) \in M_{r}(n)}^{r} \frac{n !}{k_{1} ! k_{2} ! \ldots k_{r} !} k_{1}^{k_{2}} \ldots k_{r-1}^{k_{r}}=(n+1)^{n-1} .
$$

Proof. The binomial formula reads as

$$
n^{n-k-1}=(n-k+k)^{n-k-1}=\sum_{j=1}^{n-k}\left(\begin{array}{c}
n-k \\
j
\end{array}\right) j k^{j-1}(n-k)^{n-k-j-1} .
$$

Now we proceed by induction to show that for $s \geq 1$ we have

$$
\begin{aligned}
(n+1)^{n-1}= & \sum_{r=1}^{s} \sum_{\left(k_{1}, \ldots, k_{r}\right) \in M_{r}(n)} \frac{n !}{k_{1} ! \ldots k_{r} !} k_{1}^{k_{2}} k_{2}^{k_{3}} \ldots k_{r-1}^{k_{r}} \\
& \quad+\sum_{m=s}^{n-1} \sum_{\left(k_{1}, \ldots, k_{s}\right) \in M_{s}(m)} \frac{n !}{k_{1} ! \ldots k_{s} !(n-m) !} k_{s} k_{1}^{k_{2}} \ldots k_{s-1}^{k_{s}}\left(n-m+k_{s}\right)^{n-m-1} .
\end{aligned}
$$

Having established the basic formula we immediately derive the following corollary. 
Corollary 2.2. Let $n \geq 1$. Then

$$
\sum_{r=1}^{n} \sum_{\left(k_{1}, \ldots, k_{r}\right) \in M_{r}(n)} \frac{n !}{k_{1} ! k_{2} ! \ldots k_{r} !} k_{1}^{k_{1}-1} \ldots k_{r}^{k_{r}-1}=(n+1)^{n-1} .
$$

Proof. As mentioned before the previous theorem every tree with $n+1$ labeled vertices $\left\{v_{0}, \ldots, v_{n}\right\}$ can be represented as a rooted tree with (say) root $v_{0}$. Consider a partition of $\left\{v_{1}, \ldots, v_{n}\right\}$ into subsets $E_{1}, \ldots, E_{r}$ of cardinalities $k_{1}, \ldots, k_{r}$. The number of labeled trees with vertices from $E_{l}$ is $k_{l}^{k_{l}-2}$ according to Cayley's formula. There are $k_{l}$ choices for a root in $E_{l}$. For each choice of a root in $E_{l}$ and each tree in $E_{l}, l=1, \ldots, r$, we can construct a unique tree of all vertices by connecting the roots in $E_{l}$ with $v_{0}$. This has $\prod_{l=1}^{r} k_{l}^{k_{l}-1}$ choices. Summing over $E_{1}, \ldots, E_{r}$, then over $k_{1}, \ldots, k_{r} \geq 1$ with $k_{1}+\ldots k_{r}=n$, and finally over $r=1, \ldots, n$ shows the corollary.

\section{The AVAlanche SIZE}

In this section we study avalanches in more detail. Let $N \in \mathbb{N}$. Assume that for each $i=1, \ldots, N S_{i}: X_{i} \rightarrow X_{i}$ are continuous transformations on the metric space $X_{i}$ with metrics $d_{i}$. Let $U_{i}$ be open sets such that the family $\left\{S_{i}^{-l}\left(U_{i}\right): 0 \leq l \leq M\right\}$ consists of pairwise disjoint sets, where $M>N$ is some fixed integer. We define the avalanche size in this section and determine its distribution under the product measure on $X=X_{1} \times \ldots \times X_{N}$ when the transformations $S_{i}$ are invariant for the probability measures $m_{i}$ on the Borel sets of $X_{i}$.

There are some basic examples, the reader may have in mind. The first illustrates Levina's model, the second one the ergodic case and the third one the "mixing case". All examples are invertible transformations, but the results of this section also hold for non-invertible maps.

Example 3.1. (a) Take $X_{i}=[0,1)$ and $S_{i}(x)=x+\delta$. This is a special case of Levina's model when $\alpha=\delta$. Take $U_{i}=(1-\delta, 1)$ for each $i=1, \ldots, N$ and $N=M$, and assume that $N \delta<1$. Later we also will consider invariant measures, here Lebesgue measure.

(b) Let $X_{i}=[0,1)$ and $S_{i}(x)=x+\delta_{i}$ where the $\delta_{i}$ are rationally independent. Here we take $M=N, U_{i}=\left(1-\delta_{i}, 1\right)$ and assume that $N \delta_{i}<1$ for each $i=1, \ldots, N$. For later purpose, also Lebesgue measure is invariant for each transformation $S_{i}$.

(c) Let $X_{i}=\left\{1, \ldots, n_{i}\right\}^{\mathbb{Z}}$ and $S_{i}$ the shift transformation with $2 \leq n_{i} \in \mathbb{N}$. We can take $M=N$ and a cylinder $U_{i}=\left[a_{0}, \ldots, a_{L}\right] \subset X_{i}$ with $a_{1}, \ldots, a_{N} \neq a_{0}$ and $L>N$. The invariant measure to use later will be a Bernoulli measure on each $X_{i}$.

The avalanche size is defined as follows: For $x=\left(x_{1}, x_{2}, \ldots, x_{N}\right) \in X$ define $A(x, 0)=0$ and if $k \geq 1$ let

$$
A(x, k)=\left|\left\{1 \leq i \leq N: \exists 1 \leq l \leq k: S_{i}^{l}\left(x_{i}\right) \in U_{i}\right\}\right| .
$$

Then we set

$$
A(x)=\max \{A(x, k): \forall j \leq k, A(x, j) \geq j\}
$$


Note that the sequence $A(x, k)$ is increasing. We also let

$$
k(x)=\max \{k: \forall j \leq k, A(x, j) \geq j\} .
$$

The avalanche transformation is then defined by

$$
S_{A}(x)=S^{A(x)+1}(x) .
$$

Lemma 3.2. For all $x \in X$ and $1 \leq i \leq N$ we have that

$$
S_{i}^{A(x)+1}\left(x_{i}\right) \notin U_{i} \text {. }
$$

Proof. Let $A(x)=0$, say $A(x)=A(x, k)$ for some $k \geq 0$. Since $k \leq A(x, k)=0$ we have that $k=0$. Therefore $A(x, 1)<1$ by definition and so $A(x, 1)=0$, which means that no $i \in\{1, \ldots, N\}$ satisfies $S_{1}\left(x_{i}\right) \in U_{i}$. But $S_{A}(x)=S(x)$ proving the lemma in the case $A(x)=0$.

Now let $1 \leq A(x)=A(x, k) \geq k$. Then $A(x, k+1)<k+1$ and since the sequence $A(x, l)$ is increasing, $A(x, k)=A(x, k+1)=k$ and there is no coordinate $i$ such that $S_{i}^{k+1}\left(x_{i}\right) \in U_{i}$. This means that for all $i, S_{i}^{A(x)+1}\left(x_{i}\right) \notin U_{i}$.

Lemma 3.3. Let $A(x, k), A(x)$ and $k(x)$ be as in (3.1), (3.2) and (3.3), respectively. Then

(a) $A(x)$ is finite for all $x \in X$.

(b) $k(x)+1=\min \{k: A(x, k)<k\}$.

(c) $A(x)=A(x, k(x))=k(x)$.

(d) $A(x)+1=\min \{A(x, k): A(x, k)<k\}$.

Proof. (a) Since the iterates $S_{i}^{-l}\left(U_{i}\right)$ of $U_{i}$ are pairwise disjoint for $l=0, \ldots, N<n_{i}$ the i-th coordinate can fall only at most once into $U_{i}$ for iterations up to time $N$. Therefore $A$ is bounded by $N$.

(b) Let $\widetilde{k}(x)=\min \{k: A(x, k)<k\}$. Then $A(x, j) \geq j$ for $j<\widetilde{k}(x)$, so $k(x)+1=\widetilde{k}(x)$.

(c) $A(x, j) \geq j$ for all $j \leq k(x)$ implies that $A(x) \geq A(x, k(x)) \geq k(x)$. Moreover, $A(x, k(x)+1)<k(x)+1$ implies that $A(x)<k(x)+1$ and hence $A(x)=k(x)$ Finally $k(x) \leq A(x, k(x)) \leq A(x, k(x)+1)<k(x)+1$ shows that $A(x, k(x))=$ $k(x)$.

(d) Similar to (b).

Suppose we have a partition of $\{1,2, \ldots, N\}$ into $r+1$ sets of sizes $k_{1}, \ldots, k_{r+1}$, so $k_{1}+k_{2}+\ldots+k_{r+1}=N$. We denote the sets of the partition by $I_{1}, \ldots, I_{r+1}$. Such a partition corresponds to an avalanche of size $a=k_{1}+k_{2}+\ldots+k_{r}$ : letting the coordinates (states) in $I_{1}$ be excited in the beginning, then the coordinates in $I_{2}$ until we let the coordinates in $I_{r}$ be excited; finally we denote by $I_{r+1}$ the coordinates which are never excited.

We describe the set where this happens formally. Let

$$
E_{1}=\prod_{i \in I_{1}} S_{i}^{-1}\left(U_{i}\right) \times \prod_{i \notin I_{1}} X_{i}
$$

such that for $x \in E_{1}$ the coordinates $S_{i}\left(x_{i}\right)$ of $S(x)$ with $i \in I_{1}$ belong to the sets $U_{i}$, and are excited states. 
In the second step we have excited states in $I_{2}$ if $x$ belongs to

$$
E_{2}=\prod_{i \in I_{2}} \bigcup_{l=1}^{k_{1}} S_{i}^{-1-l}\left(U_{i}\right) \times \prod_{i \notin I_{2}} X_{i} .
$$

In general we set $k_{-1}=0, k_{0}=1$ and define sets

$$
E_{l}=\prod_{i \in I_{l}} \bigcup_{j=1}^{k_{l-1}} S_{i}^{-k_{-1}-k_{0}-k_{1}-\ldots-k_{l-2}-j}\left(U_{i}\right) \times \prod_{i \notin I_{l}} X_{i},
$$

which describes the points for which the coordinates are excited in the l-th step but not before $(l=1, \ldots, r)$.

Finally we write

$$
E_{r+1}=\prod_{i \in I_{r+1}}\left[X_{i} \backslash \bigcup_{l=1}^{a+1} S_{i}^{-l}\left(U_{i}\right)\right] \times \prod_{i \notin I_{r+1}} X_{i},
$$

where $a=k_{1}+\ldots+k_{r}$ is the total number of exited states. The set $E_{r+1}$ describes those coordinates which are not excited during the avalanche.

Lemma 3.4. Let $E=E_{1} \cap E_{2} \cap \ldots \cap E_{r+1}$, then

$$
A(x)=a \quad \text { for } x \in E .
$$

Proof. By definition, for $x \in E$ and $l=1, \ldots, r$, we have

$$
I_{1} \cup I_{2} \cup \ldots \cup I_{l}=\left\{1 \leq i \leq N: \exists 1 \leq j \leq k_{0}+k_{1}+\ldots+k_{l-1} \text { s.th. } S_{i}^{j}\left(x_{i}\right) \in U_{i}\right\} .
$$

Therefore $A\left(x, k_{0}+\ldots+k_{l-1}-1\right) \geq k_{1}+\ldots+k_{l-1}$. Moreover, for $l=1, \ldots, r-1$, $A\left(x, k_{0}+\ldots+k_{l-1}\right) \geq k_{1}+\ldots+k_{l}$ because $k_{l} \geq 1$. Since $k_{0}=1$ we have that $A(x, j) \geq j$ for $j=0,1,2, \ldots, k_{0}+k_{1}+\ldots+k_{r-1}=a-k_{r}$. Finally, by definition of $E_{r+1}$,

$$
I_{1} \cup \ldots \cup I_{k_{r}}=\left\{1 \leq i \leq N: \exists 1 \leq j \leq k_{0}+k_{1}+\ldots+k_{r-1} \text { s. th. } S_{i}^{j}\left(x_{i}\right) \in U_{i}\right\},
$$

so that $A\left(x, k_{0}+\ldots+k_{r-1}+j\right) \geq a \geq j$ for $j=0, \ldots, k_{r}-1$ and $A\left(x, k_{0}+\ldots+k_{r}\right)=$ $k_{1}+\ldots+k_{r}=a<a+1=k_{0}+\ldots+k_{r}$.

The lemma shows that on $E$ we have an avalanche of size $a$ with exited states described by the sets $I_{i}, i=1, \ldots, r$, and it follows that $\{x: A(x)=a\}$ is the disjoint union of such sets. The combinatorics of the avalanche process is described by this lemma, and it depends on the structure of the Rohklin towers $S_{i}^{-k}\left(U_{i}\right)$. Since these towers are a general feature of dynamical systems it is not surprising that the distribution of the avalanche size $A$ is general depending only on the measure through their values on $U_{i}$. In the limit, as $N \rightarrow \infty$ and the Rohklin towers speep out, the distribution also becomes independent of the measures leading to a universal power law as described below. We shall use Lemma 3.4 to deduce the distribution of the avalanche size under product measures.

We assume now that there exist $S_{i}$-invariant measures $m_{i}$ on $X_{i}, 1 \leq i \leq N$. Then the product measure

$$
m=m_{1} \times m_{2} \times \ldots \times m_{N}
$$

is $S$-invariant. We have 
Lemma 3.5. Let $I_{1}, \ldots, I_{r+1}$ be as above. Then

$$
m(E)=\prod_{i \in I_{1}} m_{i}\left(U_{i}\right) \prod_{l=2}^{r} k_{l-1}^{k_{l}} \prod_{i \in I_{l}} m_{i}\left(U_{i}\right) \prod_{i \in I_{r+1}}\left(1-(a+1) m_{i}\left(U_{i}\right)\right) .
$$

If $m_{i}\left(U_{i}\right)=p$ for all $i=1, \ldots, N$, then

$$
m(E)=p^{a}(1-(a+1) p)^{N-a} \prod_{l=1}^{r} k_{l-1}^{k_{l}} .
$$

Proof. Since $m$ is a product measure

$$
m(E)=\prod_{l=1}^{r+1} \otimes_{i \in I_{l}} m_{i}\left(E_{l}\right)
$$

By definition

$$
\otimes_{i \in I_{l}} m_{i}\left(E_{l}\right)=\prod_{i \in I_{l}} \sum_{k=1}^{k_{l-1}} m_{i}\left(S_{i}^{-k-k_{-1}-\ldots-k_{l-2}}\left(U_{i}\right)\right)=\prod_{i \in I_{l}} k_{l-1} m_{i}\left(U_{i}\right)
$$

for $l=1, \ldots, r$ and

$$
\otimes_{i \in I_{r+1}} m_{i}\left(E_{r+1}\right)=\prod_{i \in I_{r+1}} m_{i}\left(X_{i} \backslash \bigcup_{k=1}^{a+1} S_{i}^{-k}\left(U_{i}\right)\right)=\prod_{i \in I_{r+1}}\left(1-(a+1) m_{i}\left(U_{i}\right)\right) .
$$

Since $I_{l}$ has cardinality $k_{l}$ we are done.

Theorem 3.6. Let $A: X \rightarrow \mathbb{N}$ denote the avalanche size. Define

$$
K_{r}(a)=\left\{\left(k_{1}, k_{2}, \ldots, k_{r+1}\right): k_{1}, \ldots, k_{r} \geq 1, k_{r+1}=N-a ; \sum_{i=1}^{r} k_{i}=a\right\} .
$$

Then for any $a=0,1,2, \ldots, N$

$$
\begin{aligned}
& m(\{x \in X: A(x)=a\})= \\
& \sum_{r=1}^{a} \sum_{\left(k_{1}, k_{2}, \ldots, k_{r+1}\right) \in K_{r}(a)} \sum_{I_{1}, \ldots, I_{r+1}} \prod_{i \in I_{1}} m_{i}\left(U_{i}\right) \prod_{l=2}^{r} k_{l-1}^{k_{l}} \prod_{i \in I_{l}} m_{i}\left(U_{i}\right) \prod_{i \in I_{r+1}}\left(1-(a+1) m_{i}\left(U_{i}\right)\right),
\end{aligned}
$$

where $\sum_{I_{1}, \ldots, I_{r+1}}$ denotes summation over all partitions of $\{1, \ldots, N\}$ into sets $I_{1}, \ldots, I_{r+1}$ of sizes $k_{1}, \ldots, k_{r+1}$.

In particular, if $m_{i}\left(U_{i}\right)=p$ for all $i=1, \ldots, N$, then

$$
m(\{x \in X: A(x)=a\})=(a+1)^{a-1}\left(\begin{array}{c}
N \\
a
\end{array}\right) p^{a}(1-(a+1) p)^{N-a} .
$$

Proof. Note that the first formula is immediate from the foregoing discussion. Moreover, if all $m_{i}\left(U_{i}\right)=p$ for some $p \in[0,1]$ then, by Theorem 2.1 , the formula reduces 
to

$$
\begin{aligned}
& m(\{x \in X: A(x)=a\}) \\
= & \sum_{r=1}^{a} \sum_{k_{1}+k_{2}+\ldots+k_{r}=a ; k_{r+1}=N-a} \frac{N !}{k_{1} ! k_{2} ! \ldots k_{r+1} !} p^{a} \prod_{l=2}^{r}\left(k_{l-1}\right)^{k_{l}}(1-(a+1) p)^{N-a} \\
= & \sum_{r=1}^{N} \sum_{k_{1}+k_{2}+\ldots+k_{r}=a} \frac{a !}{k_{1} ! k_{2} ! \ldots k_{r} !} \prod_{l=2}^{r}\left(k_{l-1}\right)^{k_{l}}\left(\begin{array}{c}
N \\
a
\end{array}\right) p^{a}(1-(a+1) p)^{N-a} \\
= & (a+1)^{a-1}\left(\begin{array}{c}
N \\
a
\end{array}\right) p^{a}(1-(a+1) p)^{N-a} .
\end{aligned}
$$

Remark 3.7. (a) Let $p=\alpha / N$. Then for each $a$

$$
\lim _{\alpha \rightarrow 1} \lim _{N \rightarrow \infty} \log \frac{m(\{A=a\})}{m(\{A=a+1\}}=-\frac{3}{2 a}+o\left(\frac{1}{a}\right) .
$$

The proof of this fact uses Taylor expansion of the logarithm:

$$
\begin{aligned}
\lim _{\alpha \rightarrow 1} \lim _{N \rightarrow \infty} \log \frac{m(\{A=a\})}{m(\{A=a+1\}} & =1+a \log \frac{a+1}{a+2} \\
& =1+a\left(-\frac{1}{a+2}-\frac{1}{2(a+2)^{2}}\right)+o(1 / a) \\
& =\frac{2}{a+2}-\frac{a}{2(a+2)^{2}}+o(1 / a) \\
& =\frac{3}{2 a}+o(1 / a) .
\end{aligned}
$$

(b) Note that this asymptotic means that

$$
m(x \in X: A(x)=a) \sim a^{-3 / 2} .
$$

Note that (1) and (2) have been observed by Levina in [6].

(c) The distribution has only local maxima for $\alpha$ close to 1, i.e. in the supercritical case (see also [6]).

(d) It is straight forward to deduce a Levina type result from Theorem 3.4. Since in neural networks it is assumed that one neuron starts to fire, we are looking at the conditional distribution that one particular neuron is firing. Conditioned on this event, there are $N-1$ neurons remaining which may form an avalanche of possible sizes $1, \ldots, N$ including the initial firing neuron. According to Theorem 3.4 the distribution is given by

$$
P(A=a)=a^{a-2}\left(\begin{array}{c}
N-1 \\
a-1
\end{array}\right) p^{a-1}(1-a p)^{N-a},
$$

where $a=1, \ldots, N$. This is almost Levina's formula in $([6])$; the power in the last factor differs.

(e) In 2002 Eurich et al. [4] proposed a probability which describes the sizes $L$ of avalanches in neural dynamics. The proportionality factor in this approach has been determined by Levina [6] in 2008. In addition she was able to determine the expectation of this distribution. The distribution of $L$ has asymptotically 
(as the number of neurons $N$ tends to $\infty$ and the internal impulse $N p \rightarrow 1$ ) the following form:

Let $N$ be a positive integer and $p \in\left[0, \frac{1}{N}\right)$. Then for $k=1,2, \ldots, N$

$$
p_{k}=P(X=k)=\frac{1-N p}{1-(N-1) p} k^{k-2}\left(\begin{array}{c}
N-1 \\
k-1
\end{array}\right) p^{k-1}(1-k p)^{N-k-1}
$$

defines a probability distribution with expectation

$$
E[X]=\frac{1}{1-(N-1) p} .
$$

The distribution in (3.6) has been named Abelian distribution in [6], see also [7].

We can get the expectation of the Abelian distribution in formula (3.7) from our theorem. We claim that

$$
\frac{1-N p}{1-(N-1) p} \sum_{k=1}^{N} k^{k-1}\left(\begin{array}{c}
N-1 \\
k-1
\end{array}\right) p^{k-1}(1-k p)^{N-k-1}=\frac{1}{1-(N-1) p} .
$$

In order to prove this, for each $x \in\left[0, \frac{1}{N}\right)$ we know from Theorem 3.4 that

$$
f(x)=\sum_{a=0}^{N}(a+1)^{a-1}\left(\begin{array}{l}
N \\
a
\end{array}\right) x^{a}(1-(a+1) x)^{N-a}=1 .
$$

Taking derivative with respect to $x$ and using $N=\frac{N(1-N x)-N x}{1-(N+1) x}$ yields

$f^{\prime}(x)=\sum_{a=0}^{N}(a+1)^{a-1}\left(\begin{array}{l}N \\ a\end{array}\right) x^{a-1}(1-(a+1) x)^{N-a-1}(a(1-N x)-N x)=0$.

Multiplying by $x$ and replacing $a$ by $b-1$ we get

$$
f^{\prime}(x)=\sum_{b=1}^{N+1} b^{b-2}\left(\begin{array}{c}
N \\
b-1
\end{array}\right) x^{b-1}(1-b x)^{N-b}((b-1)(1-N x)-N x)=0 .
$$

By formula (3.6) we have

$$
\frac{1-(N+1) x}{1-N x} \sum_{b=1}^{N+1} b^{b-2}\left(\begin{array}{c}
N \\
b-1
\end{array}\right) x^{b-1}(1-b x)^{N-b}=1 .
$$

Rearranging terms in (3.8) it follows that

$$
\begin{aligned}
& \frac{1-N x}{1-(N-1) x} \sum_{b=1}^{N+1} b^{b-1}\left(\begin{array}{c}
N \\
b-1
\end{array}\right) x^{b-1}(1-b x)^{N-b}(1-N x) \\
& =\frac{1-N x}{1-(N-1) x} \sum_{b=1}^{N+1} b^{b-2}\left(\begin{array}{c}
N \\
b-1
\end{array}\right) x^{b-1}(1-b x)^{N-b} \\
& =1 .
\end{aligned}
$$




\section{ERgodicity of AVALANCHE TRANSFORMATION}

In this section we investigate the question when the avalanche transformation $S_{A}$ : $X \rightarrow X$ has an ergodic invariant measure induced by the product measure $m$ defined in the last section. This follows once we have identified the non-wandering set of the transformation. We make the assumption that each $S_{i}$ is invertible (if one of the transformations $S_{i}$ is not invertible, some of the statements below are not correct).

For $K \geq 0$ and a subset $J \subset\{1, \ldots, N\}$ with $|J| \geq K+1$, define the set

$$
B_{J}^{K}=\left\{x=\left(x_{1}, \ldots, x_{n}\right) \in X: J=\left\{1 \leq j \leq N: x_{j} \in \bigcup_{l=0}^{K} S_{j}^{l} U_{j}\right\}\right\} .
$$

Let $\mathbb{J}_{K}=\{J \subset\{1, \ldots, N\}:|J| \geq K+1\}$ and

$$
B=\bigcup_{K=0}^{N-1} \bigcup_{\emptyset \neq J \in \mathbb{J}_{K}} B_{J}^{K}
$$

By $B^{c}$ we will denote the complement of $B$.

We show first that $S_{A}$ leaves $B^{c}$ invariant and that $S_{A}$ is the induced map of $S$ on $B^{c}$.

In the first proposition we show that no $x \in B$ is an image of any point in $B^{c}$ under the $\operatorname{map} S_{A}$ :

Proposition 4.1. Let $S_{A}$ be the avalanche transformation in (3.4) and $B$ be as in (4.1). If $x \in B$ and $y \in X$ satisfying $S_{A}(y)=x$, then $y \in B$.

Proof. We prove by induction over $K=0, \ldots, N-1$ that for all sets $J \in \mathbb{J}_{K}$ we have

$$
x \in B_{J}^{K}, y \in X, S_{A}(y)=S^{A(y)+1}(y)=x \Longrightarrow y \in B .
$$

Let $K=0$ and fix a set $J \subset\{1, \ldots, N\}$ of cardinality $\geq K+1=1$. Then $J$ contains a coordinate, say $j_{0}$, and $x \in B_{J}^{0}$ means that

$$
x_{j_{0}} \in U_{j_{0}} .
$$

By Lemma 3.2, $x$ cannot be an image of a point in $X$.

Assume now that (4.2) holds for $K-1$.

Let $J \in \mathbb{J}_{K}$ and $x \in B_{J}^{K}$. For $l=0,1, \ldots, K$ we define the sets

$$
J_{l}=\left\{j \in\{1,2, \ldots, N\}: x_{j} \in S_{j}^{l}\left(x_{j}\right)\left(U_{j}\right)\right\} .
$$

Note that the sets $J_{l}$ depend on $x$ and that $J=\bigcup_{l=0}^{K} J_{l}$.

If $J_{0} \neq \emptyset$ there is some $j$ with $x_{j} \in U_{j}$ and $x$ is not an image of any $y \in X$ by Lemma 3.2 as before. Hence it remains to show the claim for any $x \in B_{J}^{K}$ for which $J_{0}=\emptyset$. In this case, $J$ contains at least $K+1$ points which are distributed among the sets $J_{l}$ for $l=1, \ldots, K$. Hence there must be at least one set containing at least two elements, so

$$
l_{0}=\min \left\{1 \leq l \leq K: \forall 1 \leq l<l_{0}:\left|J_{l}\right|=1 \&\left|I_{l_{0}}\right| \neq 1\right\}
$$

is well defined and finite.

We distinguish now two cases, the first case applies if $\left|J_{l_{0}}\right|=0$ and the second applies if $\left|J_{l_{0}}\right| \geq 2$. In each case we show the induction step separately. 
Case $J_{l_{0}}=\emptyset$ : Consider $z=S^{-l_{0}}(x)$, then for every $j \in J$, we have that

$$
z_{j}=S_{j}^{-l_{0}}\left(x_{j}\right) \in \bigcup_{l=0}^{K} S_{j}^{l-l_{0}} U_{j}
$$

while for $j \notin J$

$$
z_{j} \notin \bigcup_{l=0}^{K} S_{j}^{l-l_{0}} U_{j}
$$

Hence,

$$
z_{j} \notin \bigcup_{l=0}^{K-l_{0}} S_{j}^{l} U_{j} .
$$

Now, if $j \in J$ there is some $l \in\{1, \ldots, K\}$ with $j \in J_{l}$.

If $l<l_{0}$ then

with $l-l_{0}<0$, and this implies that

$$
z_{j} \in S_{j}^{l-l_{0}} U_{j}
$$

$$
z_{j} \notin \bigcup_{i=0}^{K-l_{0}} S_{j}^{i} U_{j}
$$

because to the contrary one would have

$$
z_{j} \in S_{j}^{l-l_{0}} U_{j} \cap S_{j}^{i} U_{j}
$$

for some $0 \leq i \leq K-l_{0}$, and the sets $U_{j}$ and $S_{j}^{l-l_{0}-i}\left(U_{j}\right)$, where $0<i+l_{0}-l \leq$ $K-l<M$, would not be disjoint.

Note that by assumption, $l=l_{0}$ is not possible since we are considering the case $J_{l_{0}}=\emptyset$.

If $l>l_{0}$, then

$$
z_{j}=S_{j}^{-l_{0}}\left(x_{j}\right) \in S_{j}^{l-l_{0}} U_{j} \subset \bigcup_{i=0}^{K-l_{0}} S_{j}^{i} U_{j}
$$

since $l-l_{0} \geq 1$.

Thus we have shown that for $I=\left\{j \in\{1, \ldots, N\}: \exists l>l_{0}\right.$ with $\left.j \in J_{l}\right\}$, we have

$$
j \in I \Longrightarrow z_{j} \in \bigcup_{i=0}^{K-l_{0}} S_{j}^{i} U_{j}
$$

and

$$
j \notin I \Longrightarrow z_{j} \notin \bigcup_{i=0}^{K-l_{0}} S_{j}^{i} U_{j} .
$$

It follows then that

$$
z \in B_{I}^{K-l_{0}}
$$

holds with $K-l_{0}<K$ and

$$
|I|=|J|-\left(l_{0}-1\right) \geq K+2-l_{0}>K-l_{0}+1 .
$$

Hence we can apply the induction hypothesis and conclude that $z$ is not an image under $S_{A}$ of any point $y \notin B$. 
To conclude the proof in this case, assume that $x$ is an image under $S_{A}$ of some point in $y \in B^{c}$. By definition of $S_{A}$, we have

$$
x=S^{A(y)+1}(z) .
$$

Writing

$$
S^{-l_{0}}(x)=z=S^{A(y)+1-l_{0}}(y)
$$

we obtain

$$
x=S^{l_{0}}(z) .
$$

If $A(y)+1 \geq l_{0}$ then $z$ is the image of a point in $B^{c}$, contradicting the fact that we showed already that this cannot happen. Hence we must have that

$$
A(y)+1<l_{0} \text {. }
$$

Then

$$
y=S^{-l}(x)
$$

for some $l \in\left\{1, \ldots, l_{0}-1\right\}$. However, $J_{l} \neq \emptyset$, so there is some $j$ with $x_{j} \in S_{j}^{l} U_{j}$, which implies that

$$
y_{j} \in U_{j}
$$

But this is impossible since points with some coordinate in its U-set belong to $B$.

We conclude that $x$ cannot be an image under $S_{A}$ of any point in $B^{c}$, finishing the proof in the first case.

Case $\left|J_{l_{0}}\right| \geq 2$ : Assume that there is some point $y \in X$ such that $S_{A}(y)=S^{A(x)+1}(y)=$ $x$.

If $A(y)+1 \in\left\{1, \ldots, l_{0}\right\}$, then for some $j \in\{1, \ldots, N\}$ we have that

$$
x_{j} \in S_{j}^{A(y)+1} U_{j},
$$

(since $\left|J_{l_{0}}\right| \geq 1$ ) and so

$$
y_{j}=S^{-A(y)+1}\left(x_{j}\right) \in U_{j} .
$$

However, we know already that these points $y$ do not belong to $B^{c}$. We conclude therefore that $A(y)+1>l_{0}$.

We write $z=S^{-l_{0}}(x)$. Then, we have $S^{A(y)+1-l_{0}}(y)=z$, with $A(y)+1-l_{0} \geq 1$, so $z$ is in the forward orbit of $y$.

Moreover, for $1 \leq l \leq l_{0}$

$$
j \in J_{l} \Longrightarrow S^{-l}\left(x_{j}\right) \in U_{j} \Longrightarrow S^{l_{0}-l}\left(z_{j}\right) \in U_{j}
$$

(recall that $\left|J_{l}\right|=1$ for $1 \leq l<l_{0}$ and $\left|J_{l_{0}}\right| \geq 2$ ).

By definition of $S_{A}$, once we reach the iteration $S^{A(y)+1-l_{0}}(y)=z$, we have at least two coordinates $j_{1}$ and $j_{2}$ falling into the level set $U_{j_{m}}$, where $m=1,2$, respectively. In each of the successive $l_{0}-1$ iterations of $z$, we have one more coordinate falling into the level set $U_{j}$. This means one has to apply $S$ at least $2+l_{0}-1=l_{0}+1$ times, in addition, in order to reach the equality $S_{A}(y)=x$. However, to reach $x$ from $z$ by iterated application of $S$ one needs $l_{0}$ steps or, more precisely, either $l_{0}$ steps or at least $l_{0}+M$ steps. But we know that $S_{A}$ needs at most $N \leq M$ steps. Hence, we have a contradiction and we finish the proof. 
Proposition 4.2. If $x \in B^{c}$ and $S_{A}(x)=S^{A(x)+1}(x)$ is the avalanche transformation, then

$$
S^{l}(x) \in B
$$

for every l such that $1 \leq l<A(y)+1$ (if $A(x)=0$ there is no such $l$ ).

Proof. If $A(x)=0$ nothing has to be shown, so let $A(x) \geq 1$. By definition of $S_{A}$ and from the fact that $l \leq A(x)$, we get

$$
\left|\left\{1 \leq i \leq N: x_{i} \in \bigcup_{j=1}^{l} S_{i}^{-j} U_{i}\right\}\right| \geq l,
$$

or

$$
\left|\left\{1 \leq i \leq N: x_{i}^{l} \in \bigcup_{j=0}^{l-1} S_{i}^{j} U_{i}\right\}\right| \geq l
$$

This means that

$$
x^{l} \in B_{J}^{l},
$$

where

$$
J=\left\{1 \leq i \leq N: x_{i}^{l} \in \bigcup_{j=0}^{l-1} S_{i}^{j} U_{i}\right\} .
$$

By definition, $x^{l} \in B$, and the proof is finished.

Let $(Y, T)$ be a dynamical system (discrete time) and $F \subset Y$. The transformation induced on $F$ is defined by

$$
T_{F}(x)=T^{\varphi(x)}(x)
$$

where $\varphi(x)=\inf \left\{n \geq 1: T^{n}(x) \in F\right\}$. This defines a dynamical system on all points in $F$ which return to $F$ infinitely often.

Theorem 4.3. Let each $S_{i}: X_{i} \rightarrow X_{i}$ be invertible and invariant under the probability $m_{i}$ on the Borel sets $\mathcal{B}_{i}$. Then there is a probability measure $\mu$ and a Borel subset $F \in \mathcal{B}=\prod_{i=1}^{N} \mathcal{B}_{i}$ such that

(a) $F$ is forward invariant under the avalanche transformation $S_{A}$.

(b) $S_{A}: F \rightarrow F$ is a measurable bijection.

(c) $X \backslash F$ is contained in the wandering set of $S_{A}$, that is: For all $x \notin F$, there exists a $k(x) \in \mathbb{N}, k(x) \leq N$ such that $S_{A}^{k(x)}(x) \notin F$.

(d) $S_{A}$ agrees with the induced transformation $S_{F}$ on $F$.

$$
\mu(C)=\frac{m(C)}{m(F)}
$$

for all $C \in \mathcal{B} \cap F$.

$$
m(F)=1-m\left(\bigcup_{K=0}^{N-1} \bigcup_{J \in \mathbb{J}_{K}} B_{J}^{K}\right) .
$$

(g) $\mu$ is invariant under the avalanche transformation. 
Remark 4.4. The theorem shows that all models in Example 3.1 define avalanche transformations which are induced tranformations.

Proof. Consider the transformation $S_{A}$ as in (3.4). In Propositions 4.1 and 4.2 we proved that there is a set $F=B^{c}$ such that $S_{A}$ is an induced map on $F$ with respect to the product transformation $S$. This proves the first four assertions.

The product measure $m=m_{1} \times \ldots \times m_{N}$ is invariant with respect to the product transformation, so $(X, \mathcal{B}, m, S)$ is a probability preserving dynamical system. Define $\mu$ as in (4.3). It is known that $\mu$ is invariant for the induced map, so also for $S_{A}$.

Finally, we get (f) from (4.1).

Theorem 4.3 permits to formulate a criterion for ergodicity of the avalanche transformation.

Theorem 4.5. Let $\left(X_{i}, S_{i}, m_{i}\right)(1=1, \ldots, N)$ be probability preserving invertible transformations and $S=S_{1} \times \ldots \times S_{N}$ be the corresponding product transformation. If the systems $\left(X_{1}, S_{i}, m_{i}\right)$ have no common eigenvalues (of the associated unitary operators on $L_{1}\left(m_{i}\right)$ ) other than 1 and are all ergodic, then the avalanche transformation $S_{A}$ is ergodic.

Remark 4.6. The theorem shows that the avalanche transformation defined in Example 3.1 a. is not ergodic while the other two examples produce ergodic avalanche maps.

Proof. It is well known that the product transformation $S$ is ergodic under the product measure $m$ if all $S_{i},(1 \leq i \leq N)$, are ergodic and have no common eigenvalue other than 1 (see e.g. [1]). Then apply the fact that the induced measure is as well ergodic.

The expectation of the avalanche size distribution is unknown. Although we were able to derive this expectation for the Abelian distribution, we were not able to derive a closed form for the avalanche distribution in Section 3. This becomes an important issue since we easily can obtain a central limit theorem for processes defined by $S_{A}$ in the following way: Note that by definition the return time $\varphi$ to the set $F$ in Theorem 4.3 is given by the avalanche size: $\varphi=A+1$. Then the proof of the following result is standard, and therefore only sketched.

Theorem 4.7. Let $\left(X_{i}, S_{i}, m_{i}\right)(1=1, \ldots, N)$ be probability preserving invertible transformations with invariant probabilities $m_{i}$, and let $S=S_{1} \times \ldots \times S_{N}$ be the corresponding product transformation, which is assumed to be ergodic. Let $h_{i} \in L_{2}\left(m_{i}\right)$ be functions satisfying the central limit theorem in the form

$$
m_{i}\left(\left\{x \in X_{i}: \sum_{k=0}^{n-1} h_{i}\left(S^{k}(x)\right) \leq \sqrt{n} t \sigma_{i}\right\}\right) \rightarrow \Phi(t),
$$

where $\Phi$ denotes the distribution function of the standard normal distribution. Then, for the invariant probability $\mu$ of the avalanche transformation $S_{A}$, the function $H$ : $X \rightarrow \mathbb{R}^{N}$ defined by

$$
H\left(\left(x_{1}, \ldots, x_{N}\right)\right)=\sum_{k=0}^{A(x)}\left(h_{1}\left(S_{1}^{k}\left(x_{1}\right)\right), h_{2}\left(S_{2}\left(x_{2}\right)\right), \ldots, h_{N}\left(S_{N}^{k}\left(x_{N}\right)\right)\right)
$$


satisfies a multivariate central limit theorem in the form

$$
\mu\left(\left\{x \in F: \frac{1}{\sqrt{n}} \sum_{k=1}^{n} H\left(S_{A}^{k}(x)\right) \in R(r)\right\}\right)=P(R(r))
$$

where $R(r)=\left\{z \in \mathbb{R}^{N}: z_{k} \leq r_{k} \forall 1 \leq k \leq N\right\}$ and where $P$ is an $N$-variate normal distribution with expectation zero and covariance matrix

$$
\int[A+1] d m\left(\begin{array}{ccccc}
\sigma_{1}^{2} & 0 & 0 & \ldots & 0 \\
0 & \sigma_{2}^{2} & 0 & \ldots & 0 \\
\ldots & \ldots & \ldots & \ldots & \ldots \\
0 & 0 & 0 & \ldots & \sigma_{N}^{2}
\end{array}\right)
$$

Remark 4.8. The third model in Example 3.1 satisfies the conditions of this theorem if the $h_{i}$ are Hölder continuous.

Proof. Let $h=\left(h_{1}, \ldots, h_{N}\right)$. Since $m$ is a product measure, notice that for any $R(r)$ as in the theorem

$$
m\left(\left\{x \in X: \frac{1}{\sqrt{n}} \sum_{k=0}^{n-1} h\left(S^{k}(x)\right) \in R(r)\right\}\right) \rightarrow \widetilde{P}(R(r))
$$

where $\widetilde{P}$ is the $N$-variate normal distribution with expectation zero and covariance matrix

$$
\left(\begin{array}{ccccc}
\sigma_{1}^{2} & 0 & 0 & \ldots & 0 \\
0 & \sigma_{2}^{2} & 0 & \ldots & 0 \\
\ldots & \ldots & \ldots & \ldots & \ldots \\
0 & 0 & 0 & \ldots & \sigma_{N}^{2}
\end{array}\right) .
$$

Since the avalanche size $A$ is bounded, by the ergodic theorem

$$
\lim \frac{1}{n} \sum_{j=0}^{n-1} A\left(S^{j}(x)\right)+1=\int[A(x)+1] m(d x)=: \alpha \quad \text { a.e. }
$$

and in $L_{1}(m)$. Let $\varphi_{n}=\sum_{j=0}^{n-1}\left[A \circ S^{j}+1\right]$. Then

$$
\begin{aligned}
\frac{1}{\sqrt{n}} \sum_{j=0}^{n-1} H\left(S_{A}^{j}(x)\right) & =\frac{1}{\sqrt{n}} \sum_{j=0}^{\varphi_{n}(x)} h\left(S^{j}(x)\right) \\
& =\sqrt{\alpha} \frac{1}{\sqrt{n \alpha}} \sum_{j=0}^{n \alpha} h\left(S^{j}(x)\right)+\rho_{n}(x)
\end{aligned}
$$

where $\rho_{n}(x)=\frac{1}{\sqrt{n}} \sum_{j=n \alpha}^{\varphi_{n}(x)} h\left(S^{j}(x)\right)$ if $n \alpha \leq \varphi_{n}(x)$ and $\rho_{n}(x)=\frac{1}{\sqrt{n}} \sum_{j=\varphi_{n}(x)}^{n \alpha} h\left(S^{j}(x)\right)$ if $n \alpha \geq \varphi_{n}(x)$. By Chebychev's inequality and the maximal ergodic lemma, it is not difficult to show that

$$
\lim _{n \rightarrow \infty} m\left(\left\{x \in X: \frac{1}{\sqrt{n}} \sum_{j=0}^{n-1} H\left(S_{A}^{j}\right) \in R(r)\right\}\right)=P(R(r)) .
$$

It is left to show that we can replace $m$ by $\mu$ in the last equation. 
Let $\eta>0$ and $r \in \mathbb{R}^{N}$. Choose a Rokhlin set $E$ such that $S^{l}(E), 0 \leq l \leq K-1$, are pairwise disjoint sets covering $X$ up to a set of measure $\eta>0$. Define $C_{n}=\{x \in$ $\left.E: \frac{1}{\sqrt{n}} \sum_{k=0}^{n-1} H\left(S_{A}^{k}(x)\right) \in R(r)\right\}$.

Again, one easily shows that there is $n_{0} \in \mathbb{N}$ such that for $n \geq n_{0}$

$$
\left|m\left(C_{n}\right)-\frac{P(R(r))}{K}\right|<\frac{2 \eta}{K}
$$

The argument here also shows that the measures of the symmetric differences of the sets $\left\{x \in X: \frac{1}{\sqrt{n}} \sum_{j=0}^{n-1} H\left(S_{A}^{j}(x)\right) \in R(r \pm 2 \delta)\right\}$ and $\bigcup_{l=0}^{K-1} T^{l}\left(C_{n}\right)$ are bounded by $2 \eta$ for $n \geq n_{0}$.

Let $F$ be the set on which $S_{A}$ is defined (see Theorem 4.3). Let $K$ be so large that the set $X_{\epsilon} \subset X$ on which

$$
\left|\frac{1}{K} \sum_{j=0}^{K-1} \mathbb{I}_{F}\left(S^{j}(x)\right)-m(F)\right| \geq \epsilon
$$

has measure $\leq \epsilon$, where $\epsilon<\frac{\eta}{K}$. Then

$$
m\left(C_{n} \cap X_{\epsilon}\right) \geq \frac{P(R(r))-3 \eta}{K}
$$

Let $J \subset\{0,1, \ldots, K-1\}$ and define

$$
C_{n}(J)=\left\{x \in C_{n} \cap X_{\epsilon}: S^{l}(x) \in F \forall l \in J\right\} .
$$

Then

$$
\bigcup_{l=0}^{K-1} S^{l}\left(C_{n} \cap X_{\epsilon}\right)=\bigcup_{|| J|-K \mu(F)|<K \epsilon} \bigcup_{l \in J} T^{l}\left(C_{n}(J)\right) .
$$

It follows now that

$$
\begin{aligned}
& m\left(\left\{x \in F: \frac{1}{\sqrt{n}} \sum_{j=0}^{n-1} H\left(S_{A}^{j}(x)\right) \in R(r-2 \eta)\right\}\right) \\
\leq & m\left(\bigcup_{|| J|-K \mu(F)|<K \epsilon} \bigcup_{l \in J} S^{l}\left(C_{n}(J)\right)+3 \eta\right. \\
\leq & K(m(F)+\epsilon) \mu\left(C_{n}\right)+3 \eta \\
\leq & K(m(F)+\epsilon) \frac{P(R(r))+2 \eta}{K}+3 \eta \\
\leq & m(F) P(R(r))+\epsilon P(R(r))+5 \eta .
\end{aligned}
$$


Similarly,

$$
\begin{aligned}
& m\left(\left\{x \in F: \frac{1}{\sqrt{n}} \sum_{j=0}^{n-1} H\left(S_{A}^{j}(x)\right) \in R(r+2 \eta)\right\}\right) \\
\geq & m\left(\bigcup_{|| J|-K \mu(F)|<K \epsilon} \bigcup_{l \in J} S^{l}\left(C_{n}(J)\right)-3 \eta\right. \\
\geq & K(m(F)-\epsilon)\left(\mu\left(C_{n} \cap X_{\epsilon}\right)-3 \eta\right. \\
\geq & K(m(F)-\epsilon) \frac{P(R(r))-3 \eta}{K}-3 \eta \\
= & m(F) P(R(r))-\epsilon P(R(r))-6 \eta .
\end{aligned}
$$

Letting $\eta, \epsilon \rightarrow 0$ we obtain

$$
\lim _{n \rightarrow \infty} m\left(\left\{x \in F: \frac{1}{\sqrt{n}} \sum_{j=0}^{n-1} H\left(S_{A}^{j}(x)\right) \in R(r)\right\}\right)=m(F) P(R(r))
$$

which ends the proof.

\title{
REFERENCES
}

[1] J. Aaronson: An Introduction to Infinite Ergodic Theory. Amer. Math. Soc. 1997.

[2] A. Cayley: A theorem on trees. Quarterly J. Math. 23 (1889), 376-378.

[3] L.E. Clarke: On Cayley's formula for counting trees. J. London Math Soc. 33 (1958), 471-475.

[4] C.W. Eurich, J.M. Herrmann, U.A. Ernst: Finite size effects of avalanche dynamics. Phys. Rev. E 66 (2002), 066137.

[5] L. Lapicque: Recherches quantitatives sur l'excitation electrique des nerfs traitee comme une polarisation. J. Physiol. Pathol. Gen. 9, (1907), 620-635.

[6] A. Levina: A mathematical approach to self-organized criticality in neural networks. PhD Diss. 2008.

[7] A. Levina, J.M. Herrmann: The Abelian distribution. To appear: Stochastics and Dynamics 2014.

[8] J.W. Moon: Various proofs of Cayley's formula for counting trees. In: A seminar on graph theory. Eds. F. Harary with L. Beineke. Holt, Rinehart, Winston, New York, Chicago, San Francisco, Toronto, London, 1967, pp. 70-78.

[9] A. Renyi: On the enumeration of trees. In: Combinatorial structures and their applications. Proceedings of the Calgary international conference on combinatorial structures and their applications. University of Calgary, 1969. Eds.: R. Guy, H. Hanani, N. Sauer, J. Schonheim. Gordon and Breach, New York, London, Paris 1970. pp. 355-360.

\section{A Note on Avalange Distributions ${ }^{3}$}

\author{
Wenbo V. Li
}

\footnotetext{
${ }^{3}$ This is the adapted version of W.V. Li's comment on Dec. 12, 2012
} 
We follow the definitions and most of notations as in Levina (2008) for the model and make connections with the avalanche distribution discussed in this paper.

Let $n \in \mathbb{N}, \alpha \in(0,1], p=\alpha_{n}=\alpha / n$ and $\left(U_{i}\right)_{i=1}^{\infty}$ be i.i.d. uniformly distributed on $[0,1]$. We recursively construct the random sequence $\left(\xi_{k, n}\right)_{k=0}^{\infty}$ as follows:

$$
\begin{aligned}
\xi_{0, n} & =1, \quad \xi_{1, n}=\sum_{i=1}^{n} \mathbb{I}_{[1-p, 1]}\left(U_{j}\right), \\
\xi_{k+1, n} & =\sum_{j=1}^{n} \mathbb{I}_{\left[1-p \sum_{i=0}^{k} \xi_{i, n}, 1-p \sum_{i=0}^{k-1} \xi_{i, n}\right]}\left(U_{j}\right) .
\end{aligned}
$$

Lemma 4.9. (Levina 2008) The joint distribution of $\left(\xi_{1, n}, \ldots, \xi_{m, n}\right.$ is given by

$$
\mathbb{P}\left(\xi_{1, n}=k_{1}, \ldots, \xi_{m, n}=k_{m}\right)=\left(\begin{array}{c}
n \\
k_{1}, \ldots, k_{m}
\end{array}\right)\left(1-p \sum_{i=0}^{m-1} k_{i}\right)^{n-\sum_{l=1}^{m} k_{l}} \prod_{i=1}^{m}\left(p k_{i}\right)^{k_{i}}
$$

where $k_{0}=1$ and $k_{i} \geq 0$ for $1 \leq n \leq m$ for arbitrary integers $k_{1}, \ldots, k_{m}$.

The proof is by induction, more explanations are in Levina (2008), pages 106-107.

Next consider the size of the simplified phase-space model

$$
S_{n}=\sum_{i=1}^{n} \xi_{i, n}, \quad n \geq 1
$$

Proposition 4.10. The distribution of the avalanche size $S_{n}$ is given by

$$
\mathbb{P}\left(S_{n}=k\right)=(k+1)^{k-1}\left(\begin{array}{l}
n \\
k
\end{array}\right) p^{k}(1-(k+1) p)^{n-k}, \quad k=0, \ldots, n
$$

with expectation

$$
\mathbb{E} S_{n}=\sum_{i=1}^{n} \frac{n !}{(n-i) !} p^{i}
$$

The proof of this proposition is indicated: The first part is similar to the method in the paper. For the mean the representation of $S_{n}$ is used and shown by conditioning arguments that

$$
\mathbb{E} \xi_{i, n}=\frac{n !}{(n-i) !} p^{i}
$$


Once we know the answer for the mean, here is also a direct proof checking certain combinatoric identities. We have from the distribution

$$
\begin{aligned}
\mathbb{E} S_{n} & =\sum_{k=0}^{n} k P\left(S_{n}=k\right) \\
& =\sum_{k=0}^{n}\left(k(k+1)^{k-1}\left(\begin{array}{l}
n \\
k
\end{array}\right) p^{k}(1-(k+1) p)^{n-k}\right. \\
& =\sum_{k=0}^{n} k(k=1)^{k-1}\left(\begin{array}{l}
n \\
k
\end{array}\right) p^{k} \sum_{i=0}^{n-k}\left(\begin{array}{c}
n-k \\
i
\end{array}\right)(-1)^{i}(k+1)^{i} p^{i} \\
& =\sum_{i=0}^{n} \sum_{k=0}^{n-i}(-1)^{i} k(k+1)^{i+k-1}\left(\begin{array}{l}
n \\
k
\end{array}\right)\left(\begin{array}{c}
n-k \\
i
\end{array}\right) p^{k+i} \\
& =\sum_{j=0}^{n} \sum_{k=0}^{j}(-1)^{j-k} k(k+1)^{j-1}\left(\begin{array}{l}
n \\
k
\end{array}\right)\left(\begin{array}{c}
n-k \\
j-k
\end{array}\right) p^{j} .
\end{aligned}
$$

The key identity to use is

$$
\sum_{k=0}^{j}(-1)^{j-k} k(k+1)^{j-1}\left(\begin{array}{l}
n \\
k
\end{array}\right)\left(\begin{array}{l}
n-k \\
j-k
\end{array}\right)=\frac{n !}{(n-j) !} \quad j \leq n .
$$

As a polynomial for $n$ of degree $j$ we know both sides are zero for $n=0,1, \ldots, j-1$. The case $n=j$ can be found in Section 0.14 of Gradsteyn, Ryzhik, Jeffrey, Zwillinger's Table of Integrals, Series and Products, Taylor and Francis 2012.

The variance of the so called Abelian distribution can be found also. ${ }^{4}$

Mathematics Department, Pennsylvania State University, State College PA 16802, USA

E-mail address: denker@math.psu.edu

College of Engineering, Mathematics and Physical Sciences, Harrison Building, Streatham Campus, University of Exeter, North Park Road, Exeter, UK EX4 4QF

E-mail address: A.Rodrigues@exeter.ac.uk

\footnotetext{
${ }^{4}$ Apparently Wenbo knew a proof but had no time to write it up.
} 